\title{
Remote System for Monitoring Animal Models With Single-Metabolite Bio-Nano-Sensors
}

\author{
Sandro Carrara, Léandre Bolomey, Cristina Boero, Andrea Cavallini, Eric Meurville, Giovanni De Micheli, \\ Tanja Rezzonico Jost, Michele Proietti, and Fabio Grassi
}

\begin{abstract}
A novel system for remote monitoring of metabolism in an animal model is proposed in this paper. The system is obtained by integrating bio-nano-sensors to detect singlemetabolites, an electrochemical front-end made with off-the-shelf components, a radio frequency communication sub-system, and an antenna of new design. The system has been calibrated and tested for continuous monitoring of four different metabolites: glucose, lactate, glutamate, and adenosine triphosphate. Tests using animal models (mice) have been conducted to investigate tissue inflammation induced by the implanted bio-nano-sensors. These tests confirm that our system is suitable and reliable for remote monitoring of single-metabolites in experiments with animal models.
\end{abstract}

Index Terms-Animal monitoring, body sensor node, carbon nanotubes, implantable, SYST.

\section{INTRODUCTION}

$\mathbf{G}$ ENETICALLY engineered mice are highly useful models for emulating human diseases and studying disease mechanisms as well as for testing therapeutic strategies [1]. Remote monitoring during the experiments assures continuous acquisition of data on soluble mediators in animal model. Remote monitoring of blood pressure in mice has been already demonstrated including remote powering of the system [2]. Although many other physiological parameters, e.g. the viscosity [3], may be monitored by telemetry, the big challenge is the monitoring of the animal model at molecular level. Thus, it is so important to follow in time the biomarkers trend related to the disease under investigation. Continuous monitoring of humans is already in the market for glucose [4] as well as for lactate. The reliability of the technology for glucose remote monitoring has been validated up to 8 month in mice [5], [6]

Manuscript received May 31, 2012; revised August 31, 2012; accepted September 26, 2012. Date of publication December 4, 2012; date of current version January 29, 2013. This work was supported in part by the i-IronIC project, a grant from the Swiss Nano-Tera Initiative, and the Swiss National Science Foundation (NSF) under Project Sinergia CRSII2_127547/1 and Project Sino-Swiss IZLCZ2 123967. The associate editor coordinating the review of this paper and approving it for publication was Dr. Anna Grazia Mignani

S. Carrara, L. Bolomey, C. Boero, A. Cavallini, E. Meurville, and G. De Micheli are with the Swiss Federal Institute of Technology (EPFL), Lausanne CH-1015, Switzerland (e-mail: sandro.carrara@epfl.ch; leandre. bolomey@a3.epfl.ch; cristina.boero@epfl.ch; andrea.cavallini@epfl.ch; eric.meurville@epfl.ch; giovanni.demicheli@epfl.ch).

T. R. Jost, M. Proietti, and F. Grassi are with the Institute for Research in Biomedicine (IRB), Bellinzona 6500, Switzerland (e-mail: tanja.rezzonico@irb.usi.ch; michele.proietti@irb.unisi.ch; fabio. grassi@irb.usi.ch).

Color versions of one or more of the figures in this paper are available online at http://ieeexplore.ieee.org.

Digital Object Identifier 10.1109/JSEN.2012.2231670

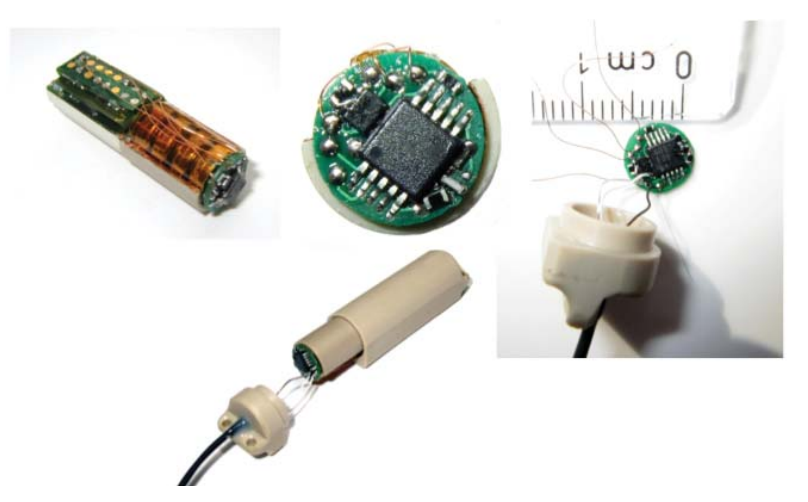

Fig. 1. System for remote acquisitions with single-metabolite bio-nanosensors.

and up to one year in pigs [7]. The next step will be the extension of the technology to other relevant metabolites like, for example, glutamate and ATP. Glutamate is an important neurotransmitter usually associated with brain damages [8] while ATP has been very recently associated to regulation of adaptive immune responses [9]. Several nano-materials have been proposed to improve devices performance including conductive polymers [10], nano-particles [11], graphene [12], and carbon nanotubes [13]. Recently, carbon nanotubes have been demonstrated to enhance the sensitivity for both exogenous [14] and endogenous [15] metabolites. The aim of the present paper is to propose the first telemetry system based on Bio-Nano-Sensors and reliable for remote and continuous single-metabolite monitoring of glucose, lactate, glutamate, and ATP in mouse models.

\section{Remote Monitoring System}

\section{A. Wireless Implantable System}

A wireless electrochemical monitoring system has been realized to assess the sensor in-vivo, as shown in Fig.1. This embedded system responds to the constraints linked to implants in animals. The materials used to build the packaging are biocompatible and support chemical sterilization process such as ethylene oxide gas or chlorine bleach. The wireless link allows us to perform measurements at distance with a laptop computer that can be easily brought into the operation room.

\section{B. Implantable Body Sensor Node}

The telemetry system is based on an implantable Body Sensor Node (BSN) for rheology monitoring, which architecture has been already described in [3], [16], [17]. 


\section{Electrochemical Front-End}

The novelty proposed in this paper for the remote monitoring system is in the electrochemical frontend. VLSI design provides fully customized solutions for also addressing multi channels chip [18] with detection of femptoampere currents [19], even including differential configurations [20]. However, the system proposed here does not require complex architectures. Therefore, our frontend to interface the BSN to the electrochemical Nano-Bio-Sensor was realized with a simple potentiostat following [21]. It is based on the commercial IC MAX4039 (manufactured by Maxim integrated products - biggest IC in the board of Fig. 1) that integrates two operational amplifiers and one voltage reference $(1.2 \mathrm{~V})$. The IC is powered by the $3 \mathrm{~V}$ battery and consumes only $9 \mu \mathrm{W}$. The potentiostat is mounted on a circular Printed Circuit Board (PCB) of $7 \mathrm{~mm}$ in diameter (photo on the right of Fig. 1), which is placed at the end of the BSN, just after the batteries. The potentiostat output is connected to the DSP analogue-todigital converter (smallest IC in the board of Fig. 1). Then the signal is low-pass filtered and the data are directly sent to the base station. A computer connected to the base station can display the data in real-time and save them for further analysis.

The computer can also set the measurement by remotely enabling and disabling the BSN. With the embedded nonrechargeable batteries exhibiting a total energy of $600 \mathrm{~J}$, the system can be powered in stand-by for over 9 years. Then, the Bio-Nano-Sensor (see Section III) is connected to a biocompatible cable in polyurethane with silver coated wires that goes through the BSN housing to the potentiostat.

\section{Single-Metabolite Bio-Nano-Sensors}

\section{A. Chemicals}

Multi-Walled Carbon Nanotubes (MWCNT) were purchased in powder (90\% purity) from Dropsens (Spain). Glucose oxidase from Aspergillus Niger (GOD, EC 1.1.3.4, 129.9 units/mg solid) lactate oxidase from Pediococcus species (LOD, EC 1.13.12.4, $\geq 20$ units/mg solid), hexokinase (HEX) type 3 from Baker Yeast, D-(+)-glucose, lithium L-lactate, and L-glutamic acid were purchased from Sigma-Aldrich (Switzerland) as lyophilized powder. Glutamate oxidase from Streptomyces species (GlOD, EC 1.4.3.11, 25 units) was supplied from Yamasa Co. (Japan). All the proteins were dissolved in Phosphate Buffer Solution (PBS) $0.01 \mathrm{M}$ at $\mathrm{pH}$ 7.4, while the other reagents were dissolved in Milli-Q.

\section{B. Screen-Printed Electrodes}

Experiments were carried out using screen-printed electrodes (SPE) from Dropsens (Spain). Working and counter electrodes are made of graphite, while the reference electrode is in $\mathrm{Ag} / \mathrm{AgCl}$. The total area of the cell is around $22 \mathrm{~mm}^{2}$, with a working electrode of $4 \mathrm{~mm}$ in diameter.

\section{Preparation of the Bio-Nano-Sensors}

A $40 \mu 1$ volume of the MWCNT-chloroform solution was deposited by drop casting ( $5 \mu 1$ each time) onto the working

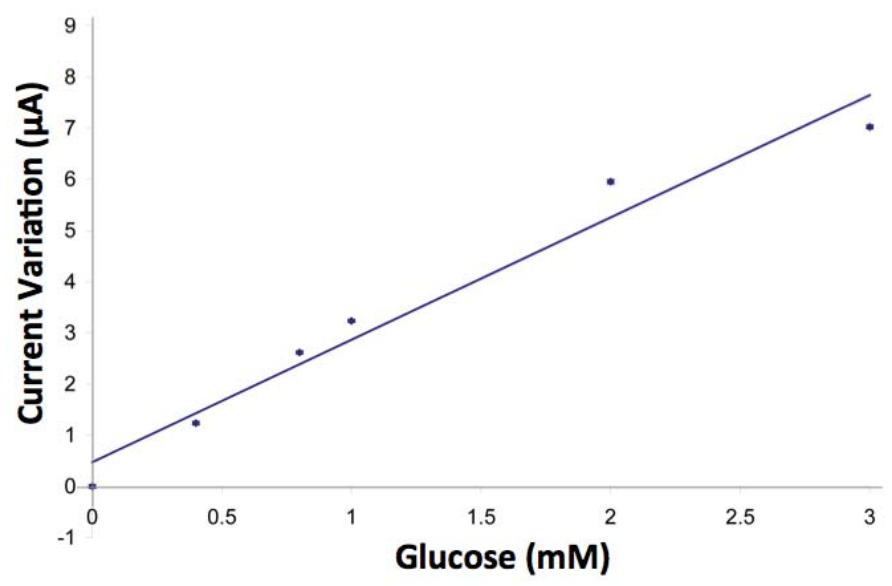

Fig. 2. Calibration curve for Glucose detection in 0 to $2 \mathrm{mM}$ concentration range.

electrode and dried. Then, $20 \mu 1$ of the enzyme probe were dropped onto the working electrode and stored overnight at $+4{ }^{\circ} \mathrm{C}$, in order to allow the adsorption of the proteins onto the electrode surface. Glucose and lactate oxidases were prepared at concentrations of $15 \mathrm{mg} / \mathrm{ml}$, and of $125 \mathrm{mg} / \mathrm{ml}$, respectively [22], [23]. The glutamate oxidase was prepared at the concentration of 250 units $/ \mathrm{ml}$. For the measure of the ATP, instead, GOD and HEX were mixed in a 1:1 ratio to obtain a solution at $\mathrm{pH} 7.4$ with $15 \mathrm{mg} / \mathrm{ml}$ of each protein [24]. $20 \mu \mathrm{l}$ of the solution were then drop cast onto the working electrode, and let dry at $4{ }^{\circ} \mathrm{C}$ overnight. The electrodes were rinsed out with Milli-Q the day after the deposition and conditioned for 10 minutes at constant potential $(+650 \mathrm{mV})$ before the first use.

\section{Calibration Curves and Telemetry Acquisitions}

For calibration and investigation of the detection limit, electrodes were dipped into the PBS with a volume of $25 \mathrm{ml}$ under stirring conditions. The electrochemical response of electrodes is investigated by chronoamperometries under aerobic conditions. The Bio-Nano-Sensors calibration has been done with the lab-electrochemical-station Versastat 3 potentiostat (Princeton Applied Technologies). After calibration, the same Bio-Nano-Sensors have been used for remote acquisitions in order to test the telemetry system described in the previous section.

\section{In-Vitro AND In-Vivo Tests}

\section{A. Calibration for Glucose, Lactate, and Glutamate}

Oxidases are enzymes that catalyze the transformation of metabolite $(X)$ in metabolism product $\left(X_{p}\right)$ by following the well-known equation:

$$
\mathrm{X}+\mathrm{O}_{2} \stackrel{\mathrm{XOD}}{\longrightarrow} \mathrm{X}_{\mathrm{P}}+\mathrm{H}_{2} \mathrm{O}_{2}
$$

The metabolite $\mathrm{X}$ is glucose, lactate, and glutamate, which are catalyzed by the glucose-oxidase (GOD), the lactate-oxidase (LOD), and the glutamate-oxidase (GLOD), respectively. 


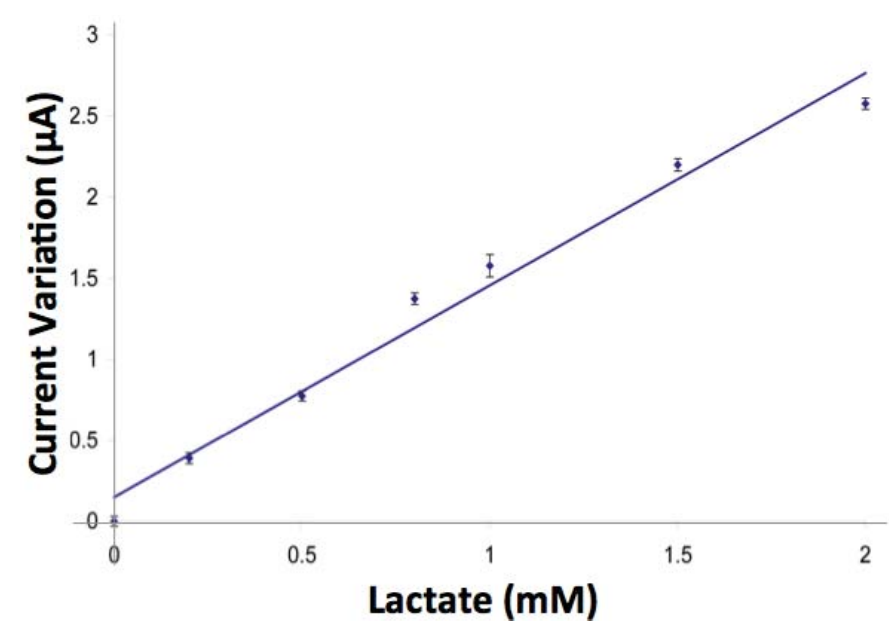

Fig. 3. Calibration curve for Lactate detection in the 0 to $1.4 \mathrm{mM}$ concentration range.

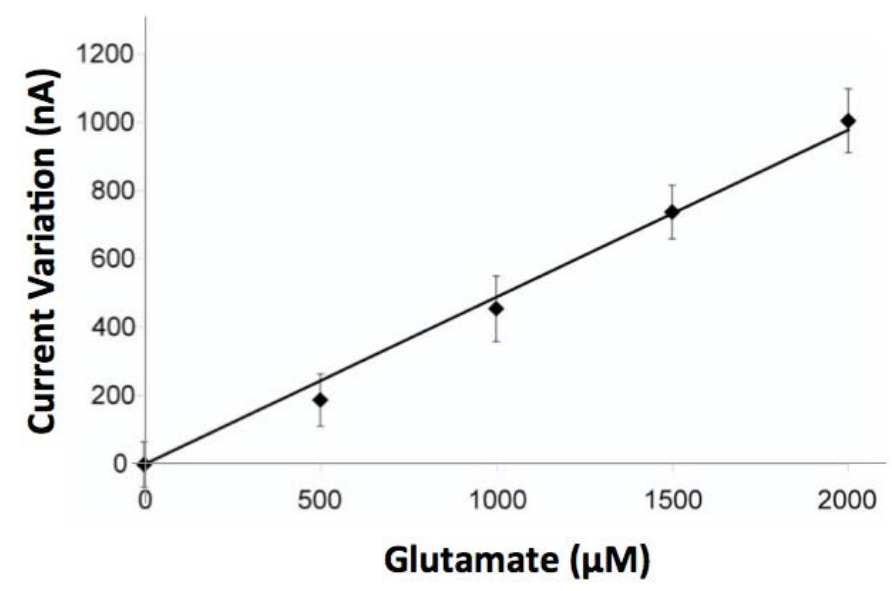

Fig. 4. Calibration curve for Glutamate detection in 0 to $2 \mathrm{mM}$ concentration range.

The reaction (1) produces the hydrogen peroxide $\left(\mathrm{H}_{2} \mathrm{O}_{2}\right)$ that may be oxidized:

$$
2 \mathrm{H}_{2} \mathrm{O}_{2} \stackrel{+650 m \mathrm{~V}}{\longrightarrow} \mathrm{O}_{2}^{+}+2 \mathrm{H}_{2} \mathrm{O}+4 e^{-}
$$

or reduced:

$$
\mathrm{H}_{2} \mathrm{O}_{2}+2 \mathrm{H}^{+}+2 e^{-+} \stackrel{1540 m V}{\longrightarrow} 2 \mathrm{H}_{2} \mathrm{O}
$$

at the interface with our electrodes. Equations (2) and (3) are two different redox reactions, the first enabled at the electrodes interface with a typical potential of $+650 \mathrm{mV}$, while the second with typical potential of $+1540 \mathrm{mV}$.

Of course, these potentials also depend on the metal of the electrodes. In presence of oxygen, reaction (2) is the most common used. So, for our Bio-Nano-Sensors we worked at $+650 \mathrm{mV}$ by following the redox of equation (2). Figs. 2-4 report the calibration curves for three different metabolites. The obtained sensitivities and the ranges of concentration are reported in Table I.

\section{B. Calibration for ATP}

In the case of the ATP monitoring, our Bio-Nano-Sensor consists of two co-immobilized enzymes: glucose oxidase and

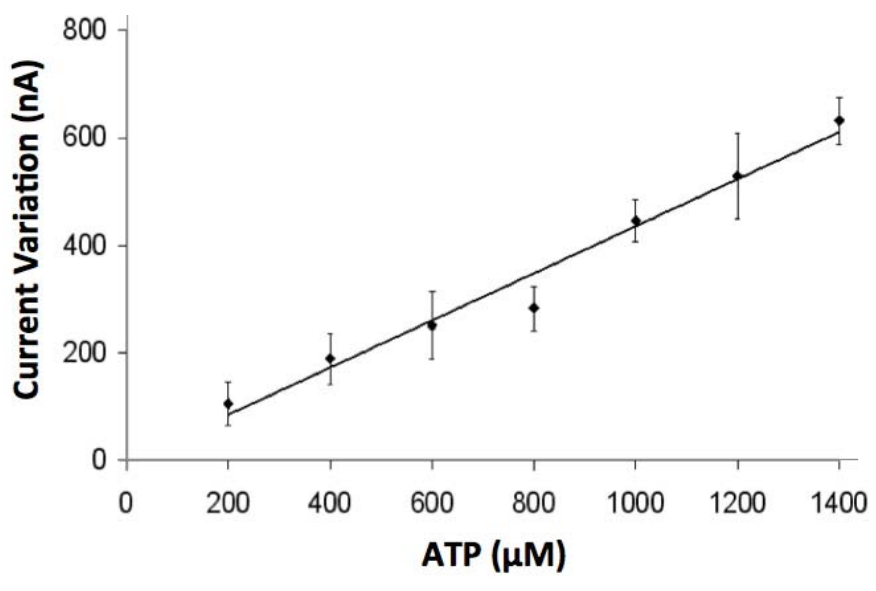

Fig. 5. Calibration curve for ATP detection in the 0 to $1.4 \mathrm{mM}$ concentration range.

TABLE I

SENSitivity AND RANGE OF THE Bio-NANO-SENSORS

\begin{tabular}{|l|l|l|}
\hline Metabolite & Sensitivity & Range \\
\hline Glucose & $299 \mathrm{nA} / \mu \mathrm{M} \mathrm{mm}^{2}$ & $0.5-4 \mathrm{mM}$ \\
\hline Lactate & $446 \mathrm{nA} / \mu \mathrm{M} \mathrm{mm}^{2}$ & $0.5-2.5 \mathrm{mM}$ \\
\hline Glutamate & $40 \mathrm{nA} / \mu \mathrm{M} \mathrm{m}^{2}$ & $0.5-2 \mathrm{mM}$ \\
\hline ATP & $34 \mathrm{pA} / \mu \mathrm{M} \mathrm{mm}^{2}$ & $200-1400 \mu \mathrm{M}$ \\
\hline
\end{tabular}

hexokinase. Both enzymes are sensitive to glucose, but with a different catalytic mechanism [24]:

$$
\begin{gathered}
D-\text { Glucose }+\mathrm{O}_{2} \stackrel{\text { GOD }}{\longrightarrow} D-\text { Gluconic Acid } \\
+\mathrm{H}_{2} \mathrm{O}_{2} . \\
\begin{aligned}
D-\text { Glucose }+A T P \stackrel{H E X}{\longrightarrow} D & - \text { Glucose }-6-P \\
& + \text { ADP. }
\end{aligned}
\end{gathered}
$$

In presence of ATP, the hexokinase competes with the glucose oxidase for the substrate, and the quantity of hydrogen peroxide produced in the reaction (4) is proportionally decreased by the glucose consumption in the reaction (5). Therefore, the ATP is detected by a decreasing of the current registered in the redox reaction (2). Fig. 5 reports the obtained calibration curve for the ATP detection The obtained sensitivity is reported in in table I, too.

\section{Telemetry Acquisitions}

The metabolite is kept by the enzymes, transformed, and then released in all the reactions involving oxidases and hexokinase. This feature of reactions (1), (4), and (5) enables continuous monitoring and acquisition over time of current variation as proportionally related to the concentration of the monitored metabolites. Fig. 6 shows the continuous monitoring of glucose over a time frame of 35 minutes, while Fig. 7 shows the continuous monitoring over a time frame of 16 minutes in the case of Lactate. Similarly, continuous monitoring of glutamate and ATP is acquired, as shown in Figs. 8 and 9. Figs. 8 and 9 clearly show that raw signals acquired on each single Bio-Nano-Sensor are typically very noisy. Noise rejection is done by averaging on 20 data points and moving this average along the entire data stream. Figs. 6 and 7 show signals after such filtering. 


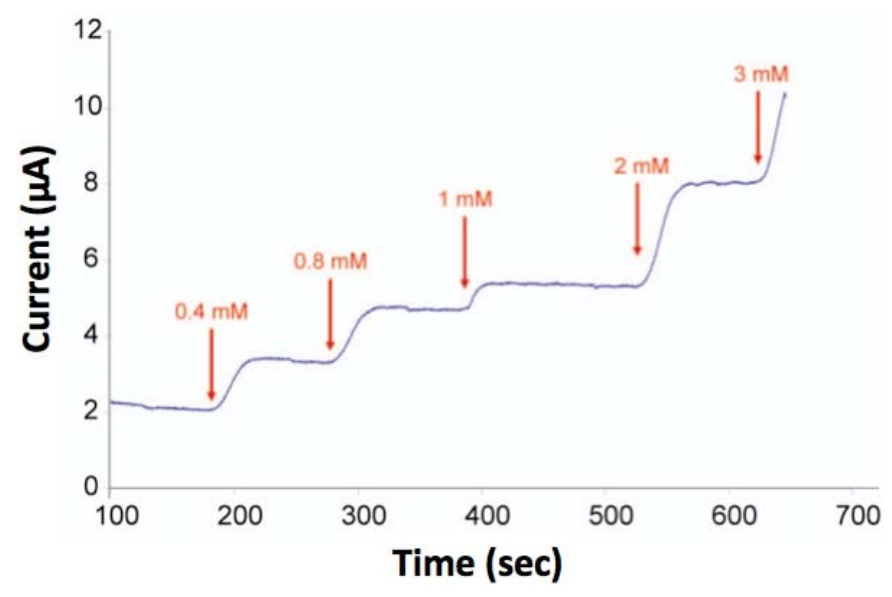

Fig. 6. Continuous monitoring with the single-metabolite remote system and a glucose bio-nano-sensor.

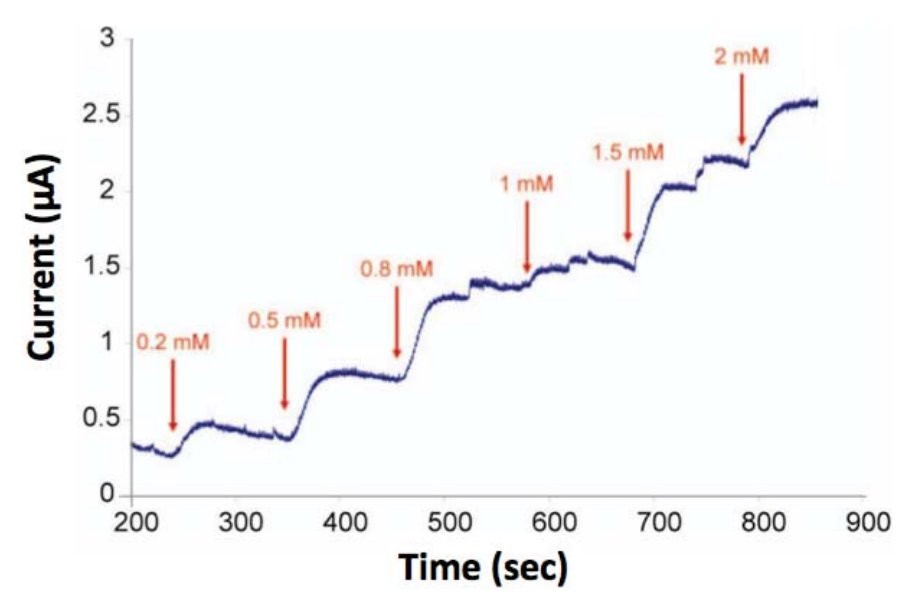

Fig. 7. Continuous monitoring with the single-metabolite remote system and a lactate bio-nano-sensor.

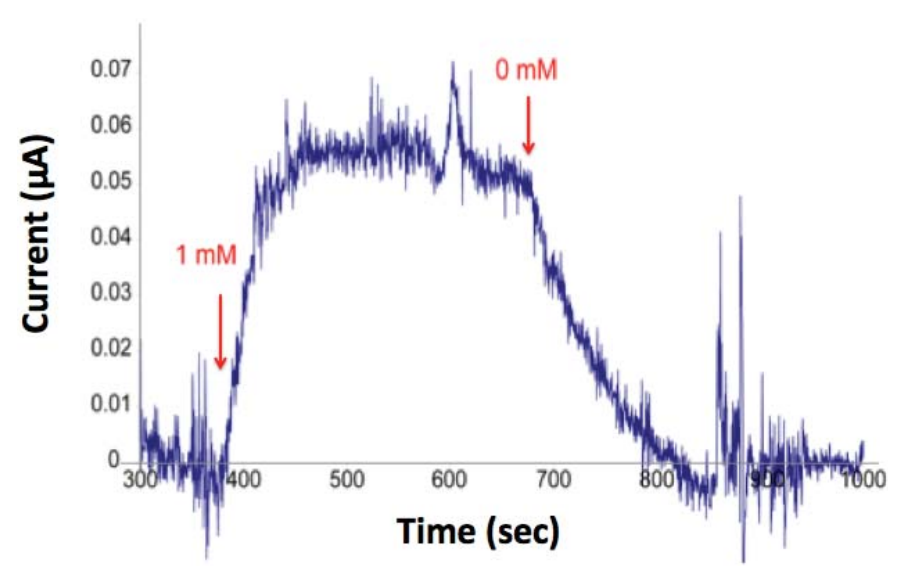

Fig. 8. Continuous monitoring with a glutamate bio-nano-sensor.

\section{Tests With Animal Models}

To check whether the Nano-Bio-Sensor has any influence on tissue homeostasis at the site of implantation, we monitored inflammatory reaction in vivo in air pouches in mice (see Fig. 10) in which the Nano-Bio-Sensors were implanted. The air pouch is generated by subcutaneous injection of sterile air into the back of a mouse. The resulting subcutaneous cavity has a diameter of around $1.5 \mathrm{~cm}$ and a height of $0.5 \mathrm{~cm}$.

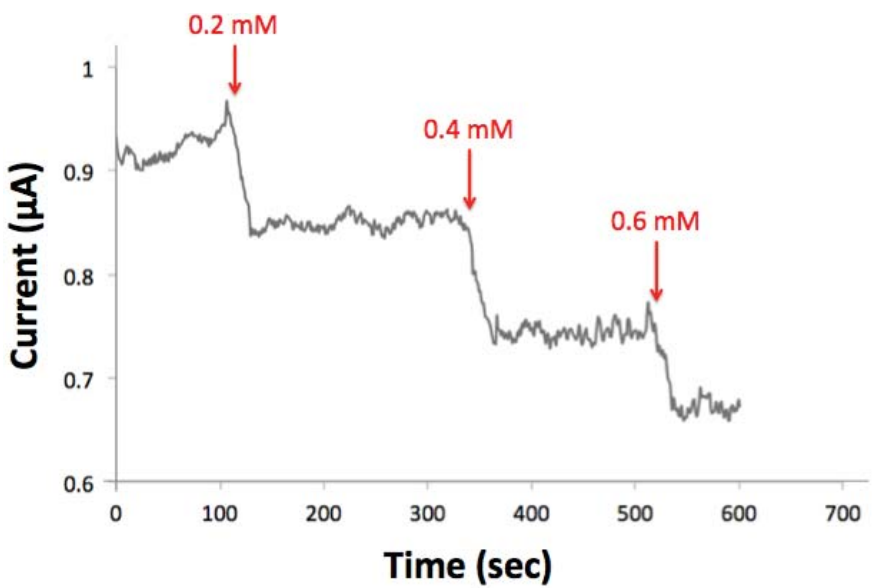

Fig. 9. Continuous monitoring with an ATP bio-nano-sensor.

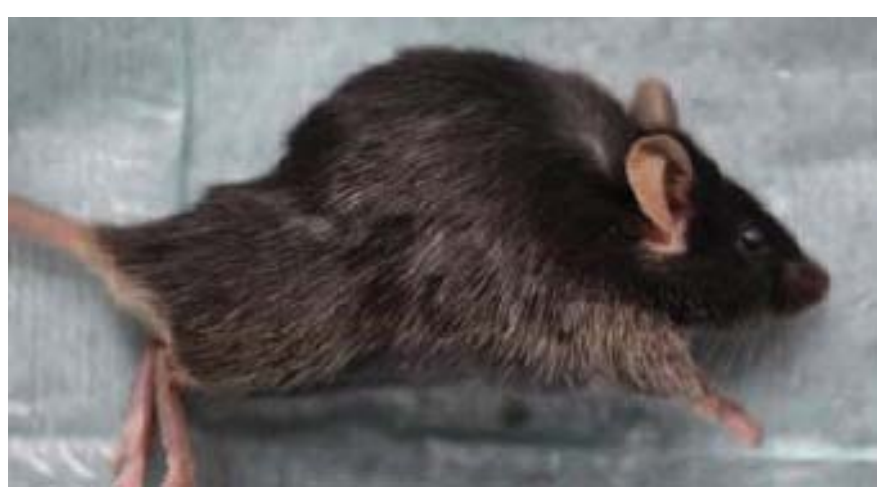

Fig. 10. Air pouch model in mice used in this paper to test the inflammatory behavior of the monitoring implants.

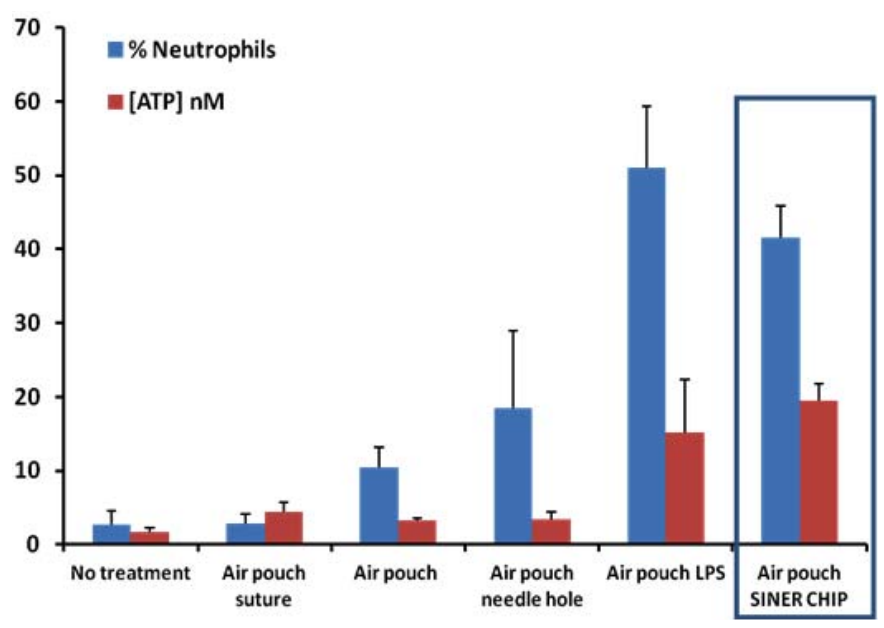

Fig. 11. Tests of inflammation in the mouse induced by the implanted bionano-sensor and the wear remote system.

The air pouch does not induce any relevant reaction of the tissue and provides an environment in which studying localized inflammatory phenomena by different stimuli, including the introduction of a Bio-Nano-Sensor. To study the impact of the implant, we inserted the sensor into the air pouch and analyzed the ATP concentration and neutrophils infiltration at day 7 after implantation. ATP is the source of energy for the cell. It is present at high concentration inside cells 
but virtually absent in extracellular fluids. Cell damage, such as the one taking place at inflammatory sites, determines the efflux of ATP from the cells and its appearance in the extracellular fluid, making it a so-called danger-associated molecular pattern. Neutrophils constitute around $50-70 \%$ of our circulating white blood cells and extravasate from blood vessels at inflammatory sites. Therefore, their presence and number is an important parameter to score inflammatory reactions. We analyzed these two parameters of inflammation in different experimental conditions. The ATP concentration in the subcutaneous microenvironment is not affected by suture. Some increase in neutrophils infiltration is detected by more invasive procedures such as performing the hole for implanting the biochip or the implantation of our Nano-Bio-Sensors.

ATP levels and neutrophils increased to a similar extent by LPS (bacterial lipopolysaccharide) injection, confirming the limited inflammation associated with sensor implantation (Fig. 11). Nevertheless, the level of ATP induced by the implant (from 20 to $60 \mathrm{nM}$ depending on different implanted sensors) is largely below the targeted range of hundreds of $\mu \mathrm{M}$ for ATP concentrations found in cell signaling [9] and relevant for anticancer treatments [25].

\section{CONClusion}

A novel system for remote monitoring of metabolism at molecular level in translational research made with animal models has been proposed in the paper. The new proposed system integrates Bio-Nano-Sensors with electrochemical frontend for the detection, a proper transceiver, battery for the powering, and a novel antenna. The Bio-Nano-Sensors are suitable for continuous monitoring of glucose, lactate, glutamate, and ATP (four highly relevant molecules of the human metabolism). Good sensitivities were provided by carbon nanotubes used as enhancers of the electrons transfer between the probe proteins and the sensors electrode.

The electrochemical front-end has been built by using outof-the-shelf components. The low-power transceiver and the antenna were especially designed for prolongation of the implantation. The tests for continuous monitoring, wireless communications, and biocompatibility demonstrated the feasibility of this technology for translational research in biomedical field with mouse models. Our in-vivo tests demonstrated mild pro-inflammatory potential at implantation site, enabling the exploitation of the proposed system for in-vivo monitoring.

\section{REFERENCES}

[1] M. Jucker, "The benefits and limitations of animal models for translational research in neurodegenerative diseases," Nature Med., vol. 16, pp. 1210-1214, Sep. 2010.

[2] P. Cong, N. Chaimanonart, W. H. Ko, and D. J. Young, "A wireless and batteryless 10-bit implantable blood pressure sensing microsystem with adaptive RF powering for real-time laboratory mice monitoring," IEEE J. Solid-Sate Circuits, vol. 44, no. 12, pp. 3631-3644, Dec. 2009.

[3] E. Meurville and P. Ryser, "Implantable ultralow power DSP-based system for a miniature chemico-rheological biosensor," Procedia Chem., vol. 1, no. 1, pp. 1235-1238, 2009.

[4] F. Valgimigli, F. Lucarelli, C. Scuffi, S. Morandi, and I. Sposato, "Evaluating the clinical accuracy of GlucoMenDay: A novel microdialysisbased continuous glucose monitor," J. Diabetes Sci. Technol., vol. 4, no. 5, pp. 1182-1192, 2010.
[5] B. Yu, N. Long, Y. Moussy, and F. Moussy, "A long-term flexible minimally-invasive implantable glucose biosensor based on an epoxyenhanced polyurethane membrane," Biosensors Bioelectron., vol. 21, no. 12, pp. 2275-2282, Jun. 2006.

[6] B. Yu, L. West, Y. Moussy, and F. Moussy, "Transcutaneous implantation methods for improving the long-term performance of glucose sensors in rats," IEEE Sensors J., vol. 8, no. 1, pp. 97-103, Jan. 2008.

[7] D. A. Gough, L. S. Kumosa, T. L. Routh, J. T. Lin, and J. Y. Lucisano, "Function of an implanted tissue glucose sensor for more than 1 year in animals," Sci. Transl. Med. vol. 2, no. 42, pp. 1-8, Jul. 2010.

[8] S. M. Rothman and J. W. Olney, "Glutamate and the pathophysiology of hypoxic-ischemic brain damage," Ann. Neurol., vol. 19, no. 2, pp. 105-111, 1986.

[9] U. Schenk, M. Frascoli, M. Proietti, R. Geffers, E. Traggiai, J. Buer, C. Ricordi, A. M. Westendorf, and F. Grassi, "ATP inhibits the generation and function of regulatory $\mathrm{T}$ cells through the activation of purinergic P2X receptors," Sci. Signal., vol. 4, no. 162, pp. 1-11, Mar. 2011.

[10] S. Paddeu, M. K. Ram, S. Carrara, and C. Nicolini, "LangmuirSchaefer films of a poly (o-anisidine) conducting polymer for sensors and displays," Nanotechnol., vol. 9, no. 3, pp. 228-232, 1998.

[11] V. Erokhin, S. Carrara, H. Amenitch, S. Bernstorff, and C. Nicolini, "Semiconductor nanoparticles for quantum devices," Nanotechnol., vol. 9, no. 3, pp. 158-162, 1998.

[12] X. Kang, J. Wang, H. Wu, I. A. Aksay, J. Liu, and Y. Lin, "Glucose Oxidase-graphene-chitosan modified electrode for direct electrochemistry and glucose sensing," Biosensors Bioelectron., vol. 25, no. 4, pp. 901-905, 2009.

[13] A. Guiseppi-Elie, C. Lei, and R. H. Baughman, "Direct electron transfer of glucose oxidase on carbon nanotubes," Nanotechnol., vol. 13, no. 5, pp. 559-564, 2002.

[14] S. Carrara, V. V. Shumyantseva, A. I. Archakov, and B. Samorì, "Screenprinted electrodes based on carbon nanotubes and cytochrome P450scc for highly sensitive cholesterol biosensors," Biosensors Bioelectron., vol. 24, no. 15, pp. 148-150, Sep. 2008.

[15] S. Carrara, A. Cavallini, V. Erokhin, and G. De Micheli, "Multi-panel drugs detection in human serum for personalized therapy," Biosensors Bioelectron., vol. 26, pp. 3914-3919, Mar. 2011.

[16] F. Merli, L. Bolomey, J.-F. Zürcher, G. Corradini, E. Meurville, and A. K. Skrivervik, "Design, realization and measurements of a miniature antenna for implantable wireless communication systems," Trans. IEEE Antennas Propag., vol. 59, no. 10, pp. 3554-3555, Oct. 2011.

[17] F. Merli, L. Bolomey, F. Gorostidi, Y. Barrandon, E. Meurville, and A. K. Skrivervik, "In vitro and in vivo operation of a wireless body sensor node," in Proc. 2nd Int. ICST Conf. Wireless Mobile Commun. Healthcare, 2011, pp. 1-8.

[18] A. Gore, S. Chakrabartty, S. Pal, and E. C. Alocilja, "A multichannel femptoampere-sensitivity potentiostat array for biosensing applications," IEEE Trans. Circuit Syst., vol. 53, no. 11, pp. 2357-2363, Nov. 2006.

[19] R. Genov, M. Stanacevic, M. Naware, G. Cauwenberghs, and N. V. Thakor, "16-channel integrated potentiostat for distributed neurochemical sensing," IEEE Trans. Circuits Syst., vol. 53, no. 11, pp. 2371-2376, Nov. 2006.

[20] M. H. Nazari and R. Genov, "A fully differential CMOS potentiostat," in Proc. IEEE Int. Symp. Circuits Syst., May 2009, pp. 2177-2180.

[21] M. M. Ahmadi and G. A. Julien, "Circuit for amperometric electrochemical sensors," in VLSI Circuits for Biomedical Applications, K. Iniewski, Ed. Norwood, MA: Artech House, 2008, pp. 309-326.

[22] C. Boero, S. Carrara, G. Del Vecchio, L. Calzà, and G. De Micheli, "Highly-sensitive carbon nanotubes-based sensing for glucose and lactate monitoring in cell culture," IEEE Trans. Nanobiol., vol. 10, no. 1, pp. 59-67, Mar. 2011.

[23] C. Boero, S. Carrara, G. Del Vecchio, L. Calzà, and G. De Micheli, "Targeting of multiple metabolites in neural cell monitored by using protein-based carbon nanotubes," Sensors Actuat. B, Chem., vol, 157, no. 1, pp. 216-224, Sep. 2011.

[24] A. Cavallini, G. De Micheli, and S. Carrara, "Comparison of three methods of biocompatible multi-walled carbon nanotubes confinement for the development of implantable amperometric ATP biosensors," Sensor Lett., vol. 9, no. 5, pp. 1838-1-1844-7, 2011.

[25] P. Pellegatti, L. Raffaghello, G. Bianchi, F. Piccardi, V. Pistoia, and F. Di Virgilio, "Increased level of extracellular ATP at tumor sites: In vivo imaging with plasma membrane luciferase," PLOS ONE, vol. 3, no. 7, pp. 2599-1-2599-9, 2008. 


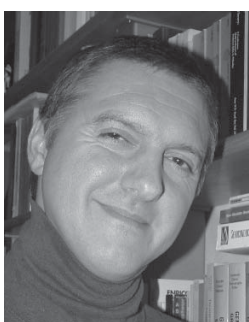

Sandro Carrara is currently a Lecturer with the Swiss Federal Institute of Technology, Lausanne, Switzerland, and a Professor of optical and electrical biosensors with the Department of Electrical Engineering and Biophysics, University of Genoa, Genoa, Italy. He has authored or co-authored more then 120 papers in journals and conferences and holds 10 patents. His current research interests include electrical phenomena of nanobiostructured films, and CMOS design of biochips based on proteins and DNA.

Dr. Carrara was a recipient of the NATO Advanced Research Award in 1996 for his original contribution to the physics of single-electron conductivity in nanoparticles, the Best Paper Award at the IEEE PRIME Conference in 2010 (Berlin) and 2009 (Cork), the Best Poster Award at the Nanotera workshop in 2011 (Bern), the Best Poster Award at the NanoEurope Symposium in 2009 (Rapperswil), and the Best Referees Award from Biosensor and Bioelectronics in 2006. He is the Founder and the Editor-in-Chief of BioNanoScience (Springer) and an Associate Editor of two IEEE journals. He is on several boards of the IEEE conferences and workshops on circuits and systems for biomedical applications.

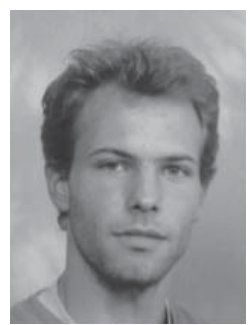

Leandre Bolomey was born in 1980 at Morges, Switzerland. He received the Masters and Ph.D. degrees in electrical sciences, with thesis entitled "Generic implantable Body Sensor Node," from the École Polytechnique Fédérale de Lausanne (EPFL), Lausanne, Switzerland, in 2003 and 2010, respectively.

He has with Xemics (Semtech) as an ASIC Test Engineer and with DspFactory (Onsemi), in 2004, where he was engaged in the design of a hearing aids analogue front-end. From 2004 to 2010, he was with the Laboratory of Microengineering for Manufacturing, EPFL, where he participated in a European project (SPARC) and was involved in the development of an embedded dual-core platform for vision applications in the automotive field, and was engaged in a CTI project, in the design of a wearable RF transceiver for a glucose sensor. In 2011, he was with an aeronautics company, where he was in charge of the validation and verification process of electronics boards for an engine monitoring unit. Since 2012, he has been with a medical company, where he is involved in the development of a system for the rehabilitation of stroke patients.

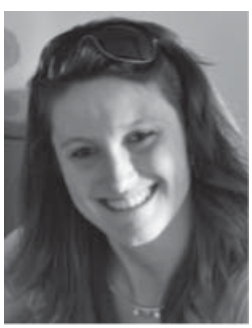

Cristina Boero received the M.Sc. degree in biomedical engineering from the Politecnico di Torino, Turin, Italy, in 2007. She is currently pursuing the $\mathrm{Ph} . \mathrm{D}$. degree with the Laboratory of Integrated Systems, École Polytechnique Fédérale de Lausanne, Lausanne, Switzerland.

Her current research interests include electrochemical biosensors for the detection of metabolites in stem cell cultures, with an aim to develop a fully integrated chip to detect such metabolites in realtime in Petri dishes, functionalization of electrodes with nanostructures, such as carbon nanotubes, and the interaction between proteins and nanostructures.

Ms. Boero was a recipient of the Gold Leaf Award at PRIME in 2009.

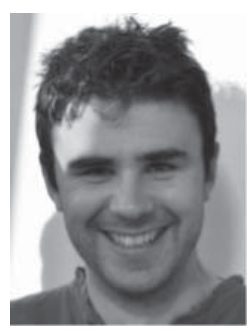

Andrea Cavallini received the Masters degree in molecular biotechnology from the University of Bologna, Bologna, Italy, and the Bachelors degree in pharmaceutical biotechnology from the University of Ferrara, Ferrara, Italy. He is currently pursuing the $\mathrm{Ph} . \mathrm{D}$. degree in microsystems and microelectronics with the École Polytechnique Fédérale de Lausanne, Lausanne, Switzerland, under the supervision of Prof. S. Carrara and Prof. G. de Micheli.

His current research interests include development of implantable biosensor arrays for personalized

therapy.

Dr. Cavallini was a recipient of the Best Poster Award at the Nanoeurope Conference in 2009 for his research on P450 biosensors, and the Bronze Leaf Award for his paper at PRIME 2010.

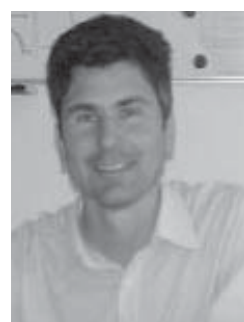

Eric Meurville received the Masters degree in electrical engineering and digital signal processing from the Conservatoire National des Arts et Métiers, Paris, France.

He has been the Head of the Product Design Group with the Laboratoire de Production Microtechnique, École Polytechnique Fédérale de Lausanne, Lausanne, Switzerland, since 1999, where he is responsible for advanced research projects on wearable and implantable biomedical devices and on the design of innovative biosensors. For the past 9 years, he has been intensively involved in research on bringing long-term implantable medical devices concepts to commercial realization. From 1995 to 1999 , he was with the Institute of Microtechnology, University of Neuchâtel, Neuchâtel, Switzerland, where he was involved in research on multimodal biometric access control systems. From 1992 to 1995, he was the Project Manager with the Laboratoire d'Etude des Transmissions Ionosphériques, Orsay, France, where he was involved in research on horizon radars. From 1986 to 1992, he was a Software and Hardware Developer of airborne electronic warfare subsystems with Thalès (formerly Dassault Electronics), France. In 2011, he co-founded gyMETRICS, whose primary aim is to bring to market easy to use noninvasive cell culture monitoring systems. This will enable improved yields and better understanding of the impact of the cell culture environment changes on cell growth

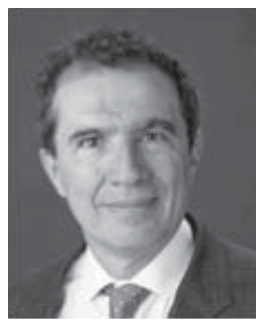

Giovanni De Micheli is currently a Professor and the Director of the Institute of Electrical Engineering and of the Integrated Systems Centre, École Polytechnique Fédérale de Lausanne, Lausanne, Switzerland. $\mathrm{He}$ is the Program Leader of the Nano-Tera.ch Program. His current research interests include several aspects of design technologies for integrated circuits and systems, such as synthesis for emerging technologies, networks on chips, 3-D integration, heterogeneous platform design including electrical components and biosensors, as well as in data processing of biomedical information.

Prof. De Micheli was a recipient of the 2003 IEEE Emanuel Piore Award for his contribution to computer-aided synthesis of digital systems, the Golden Jubilee Medal in 2000 for outstanding contributions to the IEEE CAS Society, the 1987 D. Pederson Award for the best paper at the IEEE TRANSACTIONS on CAD/ICAS, the Best Paper Award at the Design Automation Conference in 1983 and 1993, and the Best Paper Award at the DATE Conference in 2005. He is a Fellow of the ACM and a member of the Academia Europaea.

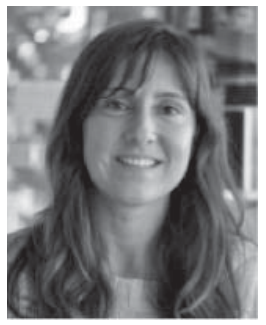

Tanja Rezzonico Jost received the Masters degree in veterinarian biotechnology sciences from the University of Milano, Milan, Italy.

She was with a veterinarian clinic Acacia, Lugano, Switzerland, from 2003 to 2010 . She is currently with the $\mathrm{T}$ Cell Development Laboratory, Institute for Research in Biomedicine, Bellinzona, Switzerland, where she is involved in research on in vivo experiments. Her current research interests include modeling human pathological conditions in mice and identifying biomarkers in chronic inflammatory

diseases.

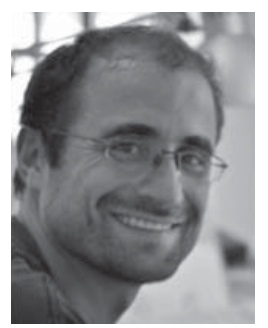

Michele Proietti received the Degree in medicine from the University of Rome, Rome, Italy, in 2001, and the Ph.D. degree in experimental and clinical immunology from the University of Genoa, Genoa, Italy.

He was a Resident with Internal Medicine, University of Rome, from 2001 to 2006, where he was involved in the management of inpatients and outpatients affected by autoimmune diseases. From 2007 to 2010, he has been a Research Fellow with the Center of Excellence for Biomedical Research, University of Genoa. He is currently a Post-Doctoral Reearcher with the T Cell Development Laboratory, Institute for Research in Biomedicine, Bellinzona, Switzerland. His curent research interests include various aspects of $\mathrm{T}$ cell physiology in inflammation and autoimmunity. 


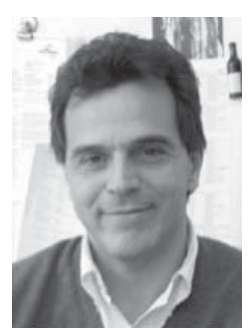

Fabio Grassi received the Degree in medicine with the University of Pavia, Pavia, Italy, and the $\mathrm{Ph} . \mathrm{D}$. degree in microbiology from the University of Milan, Milan, Italy, in 1985 and 1993, respectively.

He was an Anna Villa Rusconi Fellow with the University of Umeä, Umeä, Sweden, in 1988, a Post-Doctoral Fellow with the Institut Pasteur, Paris, France, from 1989 to 1993, an Assistant Professor with San Raffaele Scientific Institute, Milan, Italy, from 1994 to 1998, a Marie Curie Fellow with Hôpital Necker, Paris, from 1998 to 2000, and a Special Fellow of the Leukemia and Lymphoma Society with Dana Farber Cancer Institute, Harvard Medical School, Boston, MA, from 2000 to 2002. He is currently an Associate Professor of biology with the Medical School, University of Milan. In 2002, he joined the Institute for Research in Biomedicine Bellinzona, Switzerland, as the Head of the T Cell Development Laboratory. His current research interests include $\mathrm{T}$ cell physiology, including protein and membrane trafficking, signal transduction, control of cell growth and intercellular communications during $\mathrm{T}$ cell development, and immunopathological conditions. 